\title{
The Characteristics of Single Stripe Surface Dielectric Barrier Discharge with Solid Powder
}

\author{
Received March 17, 2020; revised May 14, 2020; accepted May 26, 2020
}

\author{
Yong Ho Jung ${ }^{a}$, Seungryul Yoo ${ }^{a}$, and Dong Chan Seok ${ }^{a, *}$ \\ aNational Fusion Research Institute, 37, Dongjangsan-ro, Gunsan 54004, Republic of Korea
}

*Corresponding author E-mail: stackroach@nfri.re.kr

\begin{abstract}
Surface dielectric barrier discharge (SDBD) plasma was generated with alumina powder on an SDBD electrode. Because of the higher dielectric constant of solid powder, a higher electric field is applied in the pores created by the powder particles. Depending on the powder particle sizes and gas species, the power consumption and total capacitance of the powder SDBD have characteristic trends. As the powder particle size decreases, the total volume of the discharge channels and consumed power at a given applied voltage decreased for helium (He) gas discharge; however, the opposite was observed for sulfur hexafluoride $\left(\mathrm{SF}_{6}\right)$ gas discharge at atmospheric pressure. The consumed power decreased from 82.19 to $74.5 \mathrm{~W}$ with powder diameter decrement (no powder, $1,0.1$, and $0.01 \mathrm{~mm}$ ) for He gas discharge at $10 \mathrm{kV}$ and $1 \mathrm{kHz}$ square voltage. However, for the $\mathrm{SF}_{6}$ gas discharge, the power increased from 17.86 to $49.07 \mathrm{~W}$ with $16 \mathrm{kV}$ and $1 \mathrm{kHz}$ square voltage. Therefore, the results of this study provide information regarding the characteristics of SDBD plasmas with porous dielectric media.
\end{abstract}

Keywords: Surface dielectric barrier discharge (SDBD), Powder, Glow discharge, Filament discharge

\section{Introduction}

Researchers studying plasma generally do not prefer powder materials in the plasma generation source. Solid particles in a plasma system distort the electric field and reduce the plasma conductivity, which turns off the plasma and sometimes creates fatal disasters in the vacuum facilities. Therefore, research regarding solid surface treatment using plasma has mostly focused on planar-type substrates instead of powder material, which has small volume occupation with large total surface area. However, there are numerous powder materials that could be effectively treated using plasma, such as contaminated soil and materials for manufacturing secondary batteries, crop grains, plant seeds, medicine, plastic powder, cosmetics, spices, etc. Therefore, several researchers around the world have attempted to treat powder material with their own plasma devices [1-4]. Arpagaus et al. reviewed the global studies on and industrial applications of polymer powder surface treatments using plasma [5].

A surface dielectric barrier discharge (SDBD) plasma electrode is fabricated by covering an electrically conductive layer with different shapes and areas on both sides of a dielectric plate, as shown in Fig. 1 (a). Plasma discharge can be generated on the uncovered dielectric surface through electrical breakdown of the gas near the smaller conductive layer (stripe electrode) and the high electric field by applying a high voltage between the two conductive layers. The generated discharge propagates from the edge of the small electrode toward the uncovered side on the dielectric surface, thus creating gas flow near the discharge channels. This gas flow is called electric wind. The electric wind can be used to control the flow on the wings of an airplane or to make a small size flow generator. Hence, numerous researchers have studied SDBD
[6-8].

When a thick powder layer is placed on the SDBD electrode, the electric field in the pores created by the powder particles is increased because of the higher dielectric constant of the solid particle than that of the gas species. The electric field change in the alumina powder pore is shown in Fig. 2. The FlexPDE (a commercial software) results

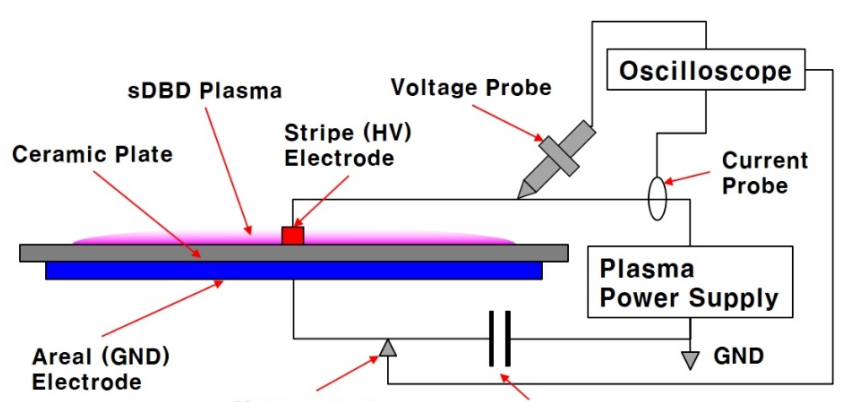

Voltage Probe Capacitor (200 nF)

(a)

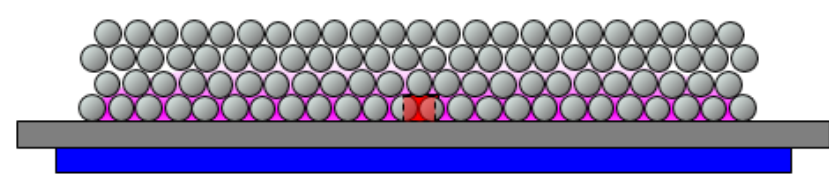

(b)

Figure 1. Experimental setup: (a) SDBD electrode structure, plasma power supply, and voltage-current-charge signal measuring connection and (b) SDBD electrode with plasma inside the powder layer. 


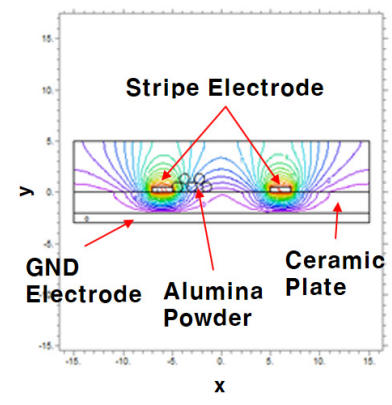

(a)

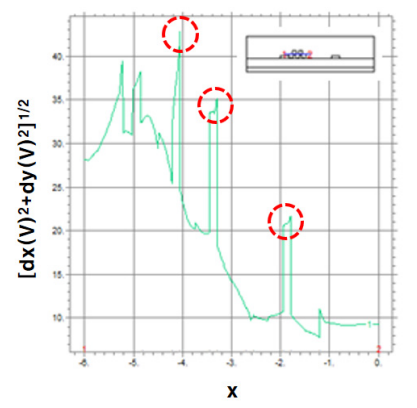

(b)
Figure 2. Static electric potential distribution ( $100 \mathrm{~V}, 1 \mathrm{~mm}$ diameter of alumina powder, $\varepsilon_{r}=9.7$ ): (a) potential distribution with the powder and (b) electric field strength in the powder pores (FlexPDE results).

and boundary conditions in Fig. 2 are not for plasma generation but for evaluating the electric field changes in the powder pores. Figure 2(a) shows five alumina particles of $1 \mathrm{~mm}$ diameter each placed around a stripe electrode of the SDBD electrode. In addition, the potential distribution of the SDBD electrode with a $100 \mathrm{~V}$ application between the stripe electrodes and ground (GND) electrode on the upper and bottom side of the ceramic plate, respectively, is shown. Figure 2(b) shows the resulting electric field change along line 1-2 across the three gaps (i.e., powder pores) created by the alumina particles, as shown in the small picture in Fig. 2(b). The electric fields in the pores steeply increase, as shown in the red dotted lines in Fig. 2(b). Therefore, the gas breakdown begins easily in the powder pores, creating plasma from the higher electric field so that the surfaces of the powder particles can be efficiently treated by the plasma in the pore. Figure 1(b) shows the gas discharge filling the pores. This study aims to introduce the discharge characteristics of SDBD with solid powder on the electrode to use the plasma source as a powder material surface treatment tool.

\section{Experimental details}

The SDBD electrode was a $125 \mathrm{~mm}$ square ceramic plate. The thickness of the plate was $1 \mathrm{~mm}$, and each side of the plate was covered with AgPd metal layers with different shapes. The material of the ceramic plate was alumina $\left(\mathrm{Al}_{2} \mathrm{O}_{3}\right)$ ceramic. A $105 \times 105 \mathrm{~mm}$ square thin metal layer, as the GND electrode, was placed on one side of the ceramic plate, and a $1 \times 105 \mathrm{~mm}$ rectangular thin metal layer, as the high voltage (HV) electrode, was placed on the center of the other side. The GND side of the electrode was cooled by recirculating water to prevent overheating of the electrode. A high voltage amplifier (20/20C HV Amplifier, TREK) combined with a function generator (33120A, Agilent) was used for the voltage source. The HV amplifier supplies 2000 times the primary input voltage (Vin) generated from the function generator into the SDBD HV electrode. The shape of the voltage used in this study was an alternating current (AC) square voltage. The displacement current of the discharge can be reduced by using square voltage. In addition, the discharge current pulse generation could be concentrated only on the short increasing/decreasing voltage phase without a memory effect of the SDBD. Helium (He) gas, which has a low breakdown voltage at atmospheric pressure, and sulfur hexafluoride $\left(\mathrm{SF}_{6}\right)$ gas, which has a high breakdown voltage, were used for plasma generation. The applied voltage and discharge current were different for the plasmas of each gas species, despite the same voltage output setup. For a comparison of the power consumption properties between the two gas discharges, Vin versus consumed power graphs were used. The points of the consumed power curves were calculated by Eq. (1) with voltage-current (V-I) oscillograms acquired for all discharge cases.

$$
P(t)=f \cdot \int_{0}^{\lambda} V(t) \cdot I(t) d t
$$

where $P(t)$ is the calculated consumed power in watts, $f$ is the voltage frequency in hertz, $\lambda$ is one cycle time of the pulse voltage in seconds, $V(t)$ is the applied voltage in volts, and $I(t)$ is the discharge current in amperes.

All the consumed power values were chosen for the average duration of three cycles. The V-I oscillograms were obtained with a high voltage probe $(\mathrm{P} 6015 \mathrm{~A}$, Tektronix, Error $= \pm 3 \%)$ and a current transformer $(110 \mathrm{~A}$, Pearson Electronics, Error $=+1 \% /-0 \%)$ and were recorded by an oscilloscope (DPO4034, Tektronix, Error $= \pm 1.5 \%$ ); therefore, the total uncertainty of the consumed power measurement was less than $\pm 4.5 \%$. Further, the accumulated charge response Q(t) was measured by insertion of a high-voltage capacitor with $200 \mu \mathrm{F}$ between the GND electrode and amplifier return terminal. Therefore, the voltage of the capacitor multiplied by the capacitance $(200 \mu \mathrm{F})$ is $\mathrm{Q}(\mathrm{t})$. The voltage probe used to measure $\mathrm{Q}(\mathrm{t})$ was $\mathrm{P} 6109 \mathrm{~B}$, manufactured by Tektronix, and the acquired $\mathrm{Q}(\mathrm{t})$ signals were used for making $\mathrm{V}-\mathrm{Q}$ Lissajous figures.

Photographs of the SDBD without powder showing one positive cycle followed by a negative voltage were obtained separately using a $0.5 \mathrm{~Hz}$ frequency voltage. The photographs were acquired with a digital camera (D700, 60 mm, F2.8, Nikon).

The powder placed on the SDBD electrode was alumina $\left(\mathrm{Al}_{2} \mathrm{O}_{3}\right)$ ceramic powder. Powders of $0.01,0.1$, and $1 \mathrm{~mm}$ particle size were used separately. The manufacturer of the powder is HANBO WORLD CO., LTD., and the product numbers of the powder are WA\#1000, WA\#150, and WA\# 16 for the $0.01,0.1$, and $1 \mathrm{~mm}$ powder, respectively. The actual size was slightly different than the nominal size. The actual sizes of the powders were $0.0131 \mathrm{~mm}( \pm 2.1 \mu \mathrm{m}), 0.0809 \mathrm{~mm}( \pm 8.4 \mu \mathrm{m})$, and $1.15 \mathrm{~mm}( \pm 0.14 \mathrm{~mm})$ for the $0.01,0.1$, and $1 \mathrm{~mm}$ powders, respectively. The particle size of the powder is the mean size of the particles, and the shape of the particle is irregular. For the powder discharge results, $100 \mathrm{cc}$ of the powder was placed on the SDBD electrode. The powder layer was flattened using a sponge and by tapping the electrode repeatedly to pack the powder. The thickness of the powder layer was $9 \mathrm{~mm}$. Because of the small pore size of the powder particles and the large thickness of the powder layer, movement of the powder particles by ionic wind was not observed. These results were evaluated using a one-side-tapered quartz prism; however, the results are not included in this paper.

\section{Results}

\subsection{Discharge at $0.5 \mathrm{~Hz}$ without powder}

Photographs and V-I oscillograms of SDBD for the positive and negative phases of the voltage were acquired separately using $0.5 \mathrm{~Hz}$ square high voltage that changes polarity every second. $\mathrm{He}$ and $\mathrm{SF}_{6}$ gas were consecutively used for the plasma generation.

The gas discharges show different appearances as the polarity 
changed. In all cases, the discharge current appeared only in the increasing and decreasing voltage periods, and there was no discharge current in the flat voltage regime. When the SDBD was generated with He gas, which has a low breakdown voltage at atmospheric pressure, the discharge channels showed glow discharge appearances beginning with numerous starting spots on the edge of the HV electrode and extending toward the dielectric surface, as shown in Figs. 3(a) and 3(b). Some of the glow discharges have relatively long and thick filament channels with glow discharge tails. The V-I oscillograms of the He gas SDBD without powder are shown in Figs. 5(a) and 6(a). In the increasing voltage phase, the discharge current shows a successive saw tooth curve. The duration of one saw tooth was $5.5 \mu$ s at the early stage and became longer, up to $8 \mu \mathrm{s}$, at the end of the increasing voltage period [Fig. 5(a)]. In the decreasing voltage phase, the discharge current shows a typical atmospheric pressure glow discharge with 5 pulse currents of micro seconds of pulse duration $[9,10]$. The duration of the pulse current was $1.5 \mu$ s at the early stage and increased to $4.5 \mu$ $\mathrm{s}$ at the end of the decreasing voltage period [Fig. 6(a)]. The different discharge currents could be easily distinguished by voltage polarity alternation; however, the photographs were difficult to differentiate. When the SDBD was generated with $\mathrm{SF}_{6}$ gas, the total size of the discharge volume was small compared to that with $\mathrm{He}$ gas. The appearance of the $\mathrm{SF}_{6} \mathrm{SDBD}$ was composed of a number of short and strong filament discharges along both edges of the HV electrode, as shown in the Fig. 4. Different from the He gas SDBD, the difference of the voltage polarity change for the $\mathrm{SF}_{6}$ gas $\mathrm{SDBD}$ without powder was obvious.

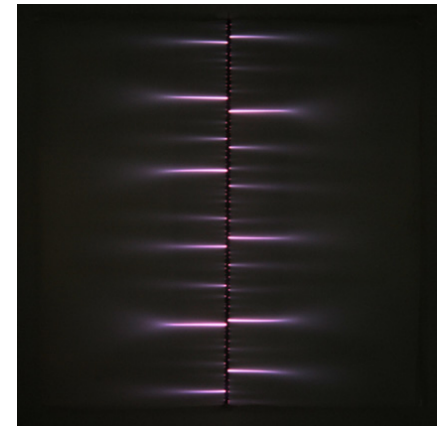

(a)

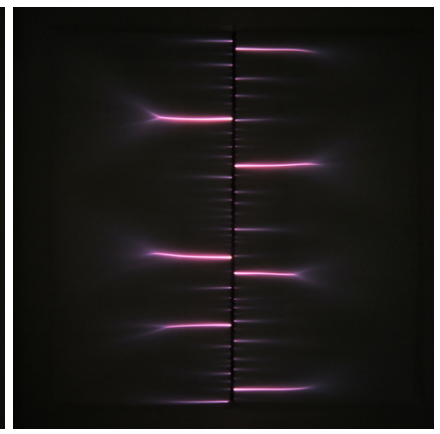

(b)
Figure 3. Photographs of the He gas SDBD discharge with $0.5 \mathrm{~Hz}$ square voltage and Vin of $5 \mathrm{~V}$ : (a) voltage rising phase and (b) voltage decreasing phase.

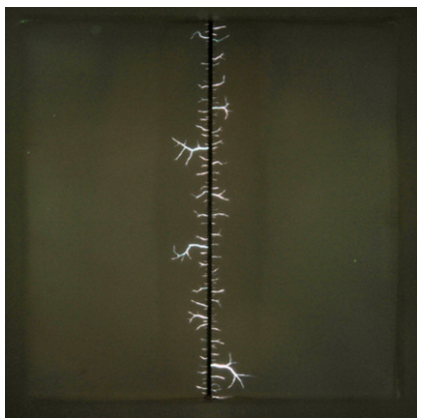

(a)

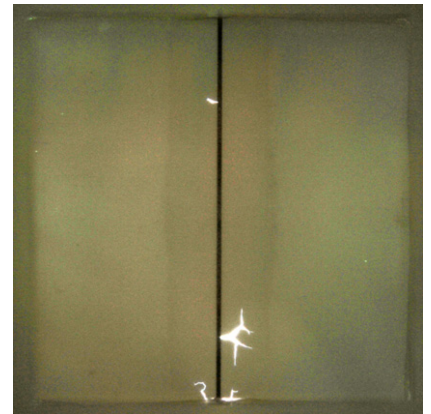

(b)
Figure 4. Photographs of $\mathrm{SF}_{6} \mathrm{SDBD}$ discharge with $0.5 \mathrm{~Hz}$ square voltage and $\mathrm{Vin}$ of $8 \mathrm{~V}$ : (a) increasing voltage phase and (b) decreasing voltage phase.
Figures 7(a) and 8(a) show the V-I oscillograms of the $\mathrm{SF}_{6} \mathrm{SDBD}$ without powder. The discharge current is composed of only pulse currents with a short duration and high peak value. Figure 7(a) shows the oscillogram of the $\mathrm{SF}_{6} \mathrm{SDBD}$ in the increasing voltage phase. The pulse currents in the increasing voltage phase are dense and have different peak values. However, the pulse currents in the decreasing voltage phase are minimal, as shown in Fig. 8(a). The shapes of the pulse currents for both polarities of the $\mathrm{SF}_{6}$ gas $\mathrm{SDBD}$ are approximately the same. When a pulse current was magnified, it had a superposition shape with a number of shorter pulses of less than $10 \mathrm{~ns}$. The tail of the pulse current had a shape of attenuating oscillation with approximately $40 \mathrm{~ns}$ of cycle time, similar to a typical atmospheric pressure filament discharge [10-12].

\subsection{Discharge at $0.5 \mathrm{~Hz}$ with powder}

Powders with a $0.01,0.1$, and $1 \mathrm{~mm}$ particle size were placed on

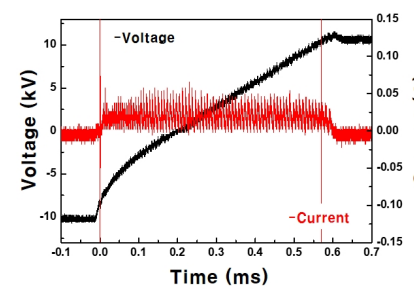

(a)

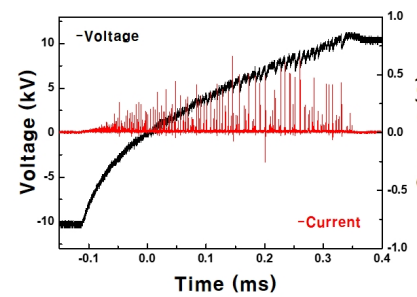

(c)

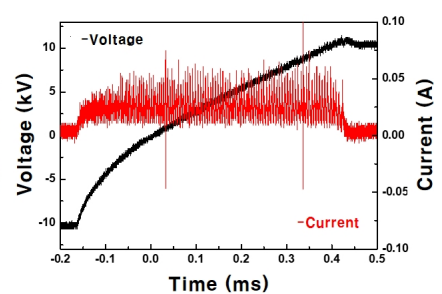

(b)

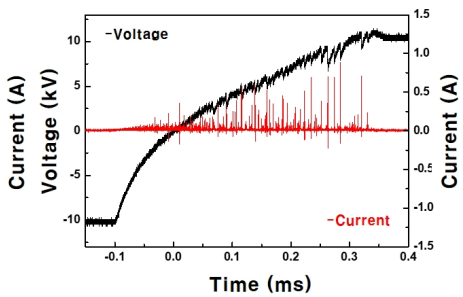

(d)
Figure 5. V-I oscillograms of the He gas powder SDBD with $0.5 \mathrm{~Hz}$ and Vin of $5 \mathrm{~V}$ in the increasing voltage phase: (a) bare, (b) $1 \mathrm{~mm}$, (c) $0.1 \mathrm{~mm}$, and (d) $0.01 \mathrm{~mm}$ size.

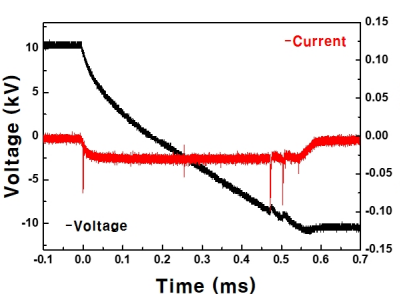

(a)

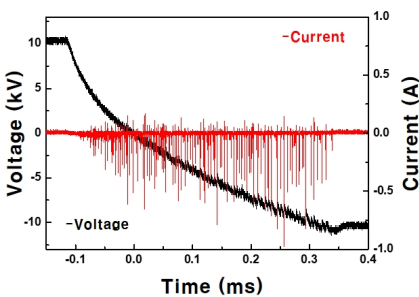

(c)

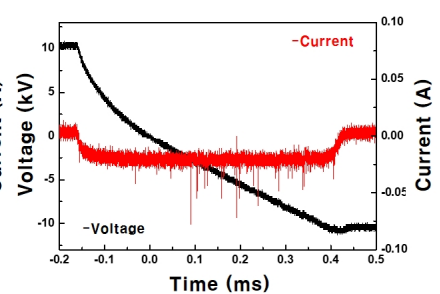

(b)

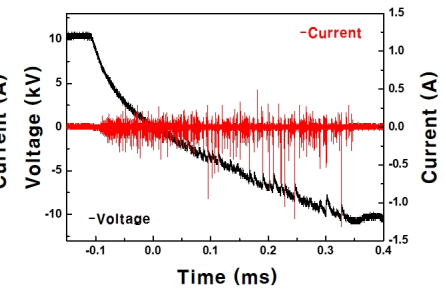

(d)
Figure 6. V-I oscillograms of the He gas powder SDBD with $0.5 \mathrm{~Hz}$ and Vin of $5 \mathrm{~V}$ in the decreasing voltage phase: (a) bare, (b) $1 \mathrm{~mm}$, (c) $0.1 \mathrm{~mm}$, and (d) $0.01 \mathrm{~mm}$ size. 


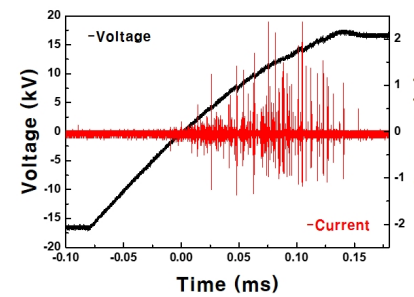

(a)

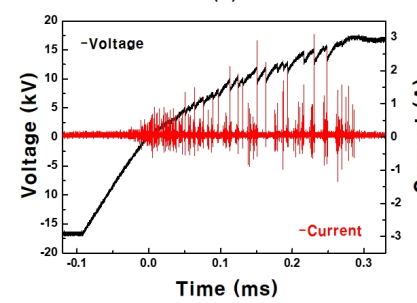

(c)

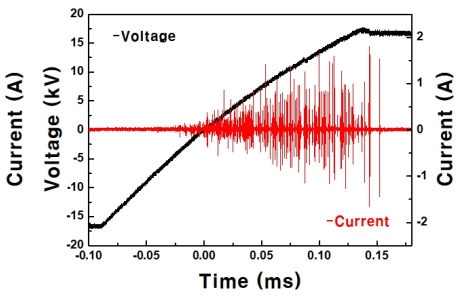

(b)

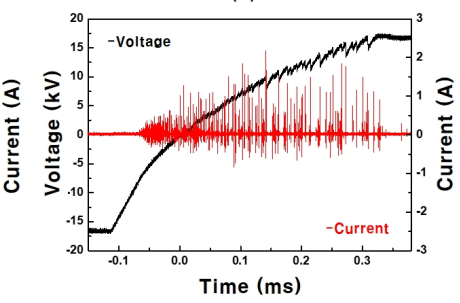

(d)
Figure 7. V-I oscillograms of the $\mathrm{SF}_{6}$ gas powder SDBD with $0.5 \mathrm{~Hz}$ and Vin of $8 \mathrm{~V}$ in the increasing voltage phase: (a) bare, (b) $1 \mathrm{~mm}$, (c) $0.1 \mathrm{~mm}$, and (d) $0.01 \mathrm{~mm}$ size.

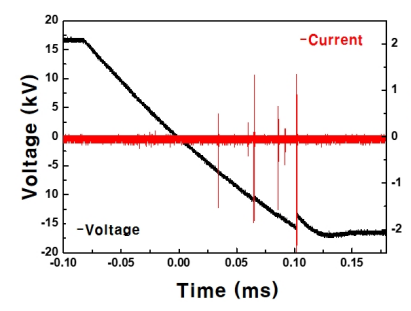

(a)

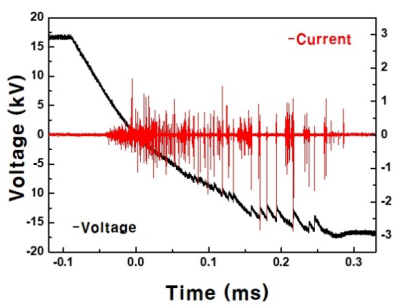

(c)

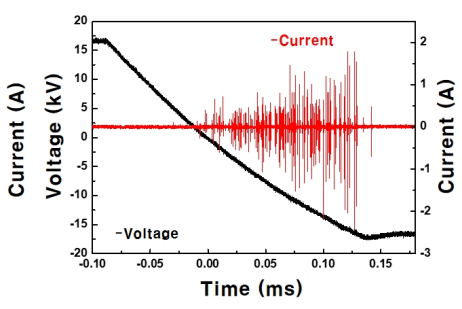

(b)

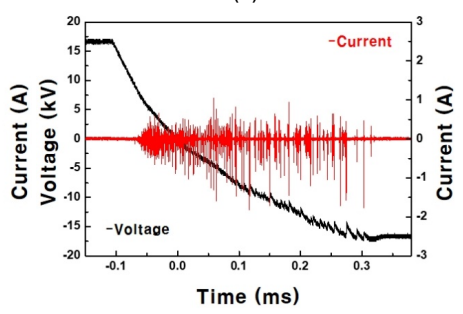

(d)
Figure 8. V-I oscillograms of the $\mathrm{SF}_{6}$ gas powder SDBD with $0.5 \mathrm{~Hz}$ and $\mathrm{Vin}$ of $8 \mathrm{~V}$ in the decreasing voltage phase: (a) bare, (b) $1 \mathrm{~mm}$, (c) $0.1 \mathrm{~mm}$, and (d) $0.01 \mathrm{~mm}$ size.

the SDBD at a $9 \mathrm{~mm}$ thickness, and the same voltages as noted in section 3.1 were applied to the SDBD electrode. Because of the thick powder layer, the generated discharge between the powder layer and SDBD electrode surface was not shown over the powder top surface. Therefore, the photographs shown in Figs. 3 and 4 were difficult to acquire for these cases. However, the existence and properties of the discharges could be identified by the V-I oscillograms based on the results given in section 3.1.

There were changes in the discharge currents of the $\mathrm{He}$ gas SDBD when the powder was placed on the SDBD electrode. Figures 5 and 6 show the discharge currents of the He gas SDBD in the increasing and decreasing voltage phases, respectively, with the powders on the SDBD. When the $1 \mathrm{~mm}$ alumina powder was placed on the SDBD electrode, the discharge current of the SDBD shows the same appearances as those without powder for both polarities. However, the frequency of the successive saw tooth in the increasing

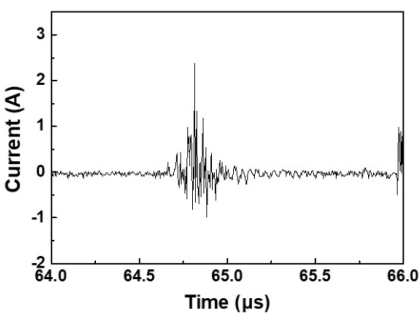

(a)

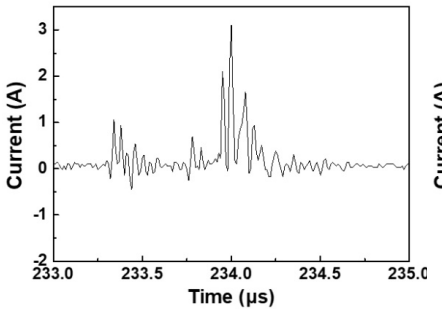

(c)

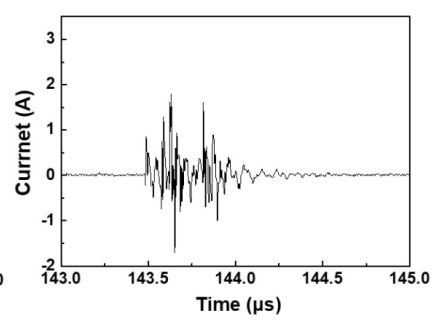

(b)

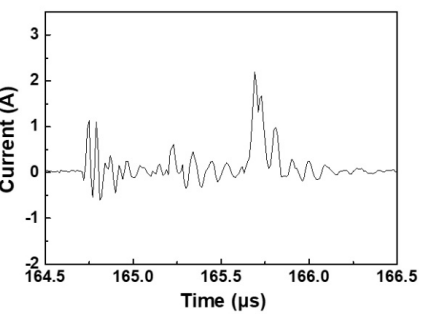

(d)
Figure 9. Magnified one pulse current of the $\mathrm{SF}_{6}$ gas powder $\mathrm{SDBD}$ with $0.5 \mathrm{~Hz}$ and Vin of $8 \mathrm{~V}$ in the increasing voltage phase: (a) bare, (b) $1 \mathrm{~mm}$, (c) $0.1 \mathrm{~mm}$, and (d) $0.01 \mathrm{~mm}$ size.

voltage phase [Fig. 5(b)] and the number of pulse currents in the decreasing voltage phase [Fig. 6(b)] increased slightly. For the 0.01 and $0.1 \mathrm{~mm}$ particle size powders, the current shapes were converted to numerous pulse currents with a short pulse duration and high peak value instead the glow discharge that was shown in the SDBD with bare and $1 \mathrm{~mm}$ powder [Figs. 5 and 6]. In the 0.01 and $0.1 \mathrm{~mm}$ powder SDBDs, the shape of one pulse current had 100 300 nanoseconds of pulse duration with a high peak value followed by an attenuating oscillation tail, as shown in a typical filament discharge [12]. This discharge regime change appeared to come from the elevated electric field in the pores made by the smaller powder [13]. The number densities and peak values of the pulse current of the 0.01 and $0.1 \mathrm{~mm}$ powder SDBDs were approximately the same for both polarities, while the pulse duration of the $0.01 \mathrm{~mm}$ powder SDBD was smaller than that of the others [Figs. 5(c), 5(d) and 6(c), 6(d)], implying that the longer filament discharge length was similar in strength to the $0.1 \mathrm{~mm}$ powder SDBD.

In the $\mathrm{SF}_{6}$ gas powder $\mathrm{SDBD}$, the voltage point at which the first pulse current appears in one phase was lowered by using the small particle size powder, as shown in Figs. 7 and 8. In addition, in the decreasing voltage phase, the number density of the pulse current increased as the particle size of the powder decreased [Fig. 8]. The shapes of the pulse currents of the 0.1 and $0.01 \mathrm{~mm}$ powder SDBDs were different from those of the bare and $1 \mathrm{~mm}$ powder SDBDs when the pulse currents were magnified, as shown in Fig. 9. In section 3.1, it was noted that the shape of a pulse current of the bare SDBD showed a pulse of short primary pulses of less than $10 \mathrm{~ns}$, followed by a vibrational attenuation tail, which was the same as that of the $1 \mathrm{~mm}$ powder SDBD [Fig. 9(b)]. However, the duration of the primary pulses of the 0.1 and $0.01 \mathrm{~mm}$ powder SDBDs increased to approximately 50 ns, as shown in Figs. 9(c) and 9(d). The properties of the superposition of the pulses and vibrational attenuation tail are the same for all $\mathrm{SF}_{6}$ gas SDBDs, regardless of the powder. Wang et al. reported that the propagation velocities of a filament discharge channel were the same when the gas species and applied voltages were 
the same $[14,15]$. Thus, placing the small size particle powder on the SDBD electrodes can lengthen the filament channels of the SDBD and facilitate the discharge start. The polarity of the voltage did not influence the duration of the pulse current of the powder SDBD.

\subsection{Power consumption characteristics for $1 \mathrm{kHz}$ frequency SDBD with powder}

The effect of the powder on the power consumption and V-Q Lissajous figure of the SDBD was examined. A stable V-Q Lissajous figure was difficult to obtain at $0.5 \mathrm{~Hz}$. Further, the calculated power values were low with the I-V signals taken at $0.5 \mathrm{~Hz}$ SDBD. Jiang et al. reported that the voltage frequency had minimal effect on the SDBD characteristics [16]. Therefore, we chose a $1 \mathrm{kHz}$ square voltage to create the SDBD for studying the power consumption characteristics and VQ Lissajous figures.

Figure 10 shows the SDBD plasmas of (a) He gas and (b) $\mathrm{SF}_{6}$ gas with a $1 \mathrm{kHz}$ square high voltage. The $\mathrm{HV}$ amplifier input voltage was 5 and $8 \mathrm{~V}$ for the $\mathrm{He}$ and $\mathrm{SF}_{6}$ gas, respectively. Unlike the $0.5 \mathrm{~Hz}$ voltage $\mathrm{He}$ gas SDBD, the $1 \mathrm{kHz}$ He gas SDBD showed uniform glow discharge covering the entire dielectric surface area of the electrode. Conversely, $1 \mathrm{kHz} \mathrm{SF}_{6}$ gas SDBD showed filamentary discharge of multiplication of the filaments that were shown in the $\mathrm{SF}_{6}$ gas SDBD with $0.5 \mathrm{~Hz}$ voltage. The input voltage to the $\mathrm{HV}$ amplifier was increased step by step to investigate the power consumption property with the applied voltage variation. The consumed power values were calculated by Eq. (1) with the recorded oscillograms at each discharge condition. The calculated consumed power was plotted to a consumed power graph according to the Vin. In all discharge cases, the graphs had the same appearances with different phase changing points. Before the discharge start, the line showed a Vin increment without a consumed power increment. After the discharge start, the consumed power increased slowly as the Vin increased, and finally, the discharge converted to where the consumed power values steeply increased without a Vin increment. The effect of the powder and size of the powder particle on the Vin-consumed power showed opposite trends for the $\mathrm{He}$ gas and $\mathrm{SF}_{6}$ gas SDBDs. For the He gas discharge case, the SDBD without powder had the lowest power with increasing Vin (temporarily PSRV), and it increased as the particle size decreased by $1,0.1$, and $0.01 \mathrm{~mm}$ sequentially with minimal difference, as shown in Fig. 11(a).

For the $\mathrm{SF}_{6}$ gas discharge, the 0.1 and $0.01 \mathrm{~mm}$ powder SDBDs

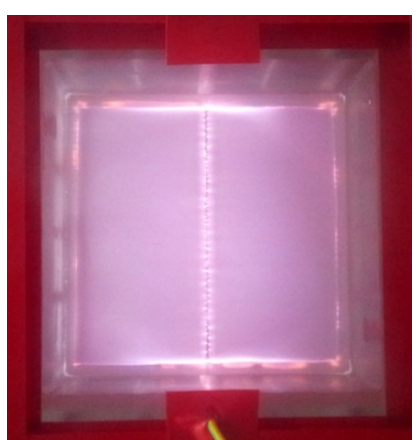

(a)

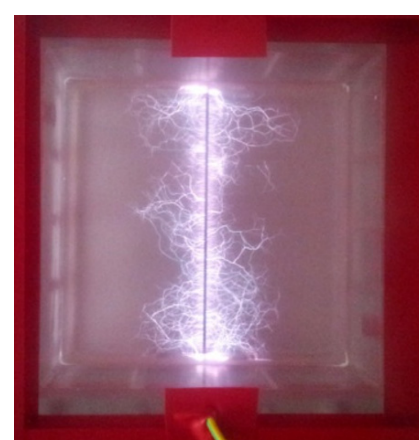

(b)
Figure 10. SDBD discharge without powder, $1 \mathrm{kHz}$ square high voltage: (a) He and (b) $\mathrm{SF}_{6}$. showed a lower PSRV with minimal difference, and the bare and 1 mm powder SDBDs showed a higher PRSV, as shown in Fig. 11(b). The effect of the powder on the Vin versus consumed power characteristics was also shown in the capacitance change by the plasma discharge on the SDBD electrode. Manley et al. introduced a method using the voltage-charge Lissajous figure (V-Q Lissajous figure) to measure the net power and capacitance of a dielectric barrier discharge (DBD) electrode with and without plasma discharge [17]. In this study, all the values of the measured power from the V-Q Lissajous figures were slightly higher than the values from Eq. (1); however, the shapes and trends of the Vin versus consumed power curves as the powder particle size changed was the same. Some studies have investigated the SDBD by the V-Q Lissajous figure method $[8,18]$. The V-Q Lissajous figure of an SDBD, which has a pair of conductive electrodes with different shapes and areas, shows an almond-like shape instead of a parallelogram for a normal DBD. However, an SDBD also shows a parallelogram Lissajous figure when the applied power is enough to saturate the discharge occupying the whole area [16].

For the He gas SDBD Lissajous figure, the differences that came from the powder and powder particle size were minimal [Fig. 12(a)]. Conversely, the shapes of the Lissajous figures of the 0.01 and $0.1 \mathrm{~mm}$ powder SDBDs showed larger enclosed areas and steeper lines for both vertical lines of the parallelogram than those of the Lissajous figures of the $1 \mathrm{~mm}$ and no-powder SDBDs. In Fig. 12(a), the slope of the straight line "a," which is parallel to the line approaching to the top right of the Lissajous figure represents the total capacitance $(\mathrm{Ct})$ of the SDBD electrode with plasma in the increasing voltage phase, and the slope of the straight line " $\mathrm{b}$ " represents $\mathrm{Ct}$ in the decreasing

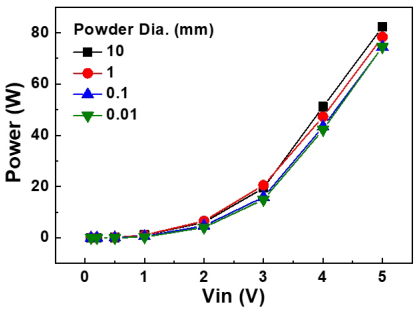

(a)

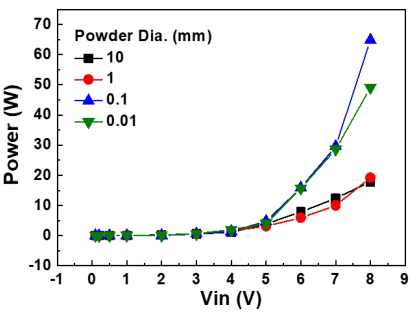

(b)
Figure 11. Voltage amplifier input voltage (Vin) to consumed power of the powder SDBD with powder particle size variation and $1 \mathrm{kHz}$ square voltage: (a) He gas and (b) $\mathrm{SF}_{6}$ gas (square 10: without powder; circle: $1 \mathrm{~mm}$; triangle: $0.1 \mathrm{~mm}$; inverted triangle: $0.01 \mathrm{~mm}$ of the powder particle size).

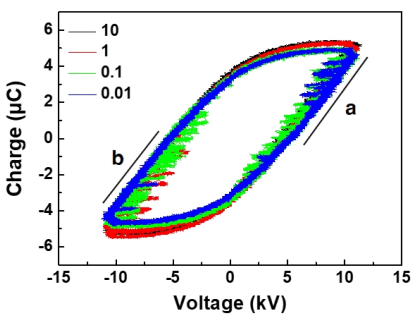

(a)

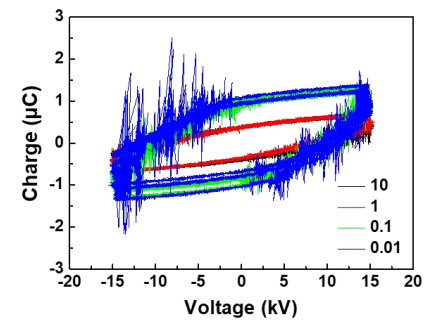

(b)
Figure 12. V-Q Lissajous figures of the powder SDBD of He gas and $\mathrm{SF}_{6}$ gas with powder particle size variation and $1 \mathrm{kHz}$ square voltage: (a) $\mathrm{He}$ and (b) $\mathrm{SF}_{6}$ (black 10: without powder; red: $1 \mathrm{~mm}$; green: $0.1 \mathrm{~mm}$; blue: $0.01 \mathrm{~mm}$ of the powder particle size). 


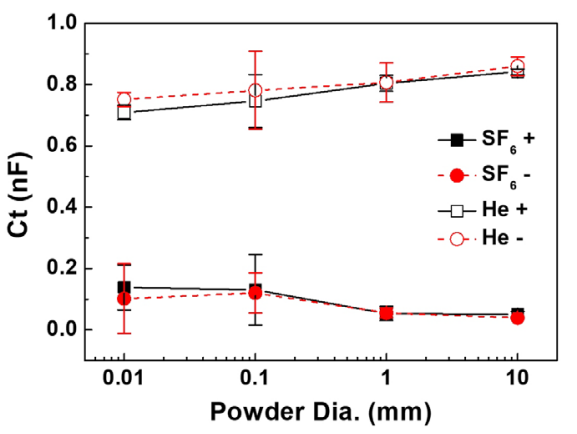

Figure 13. Total capacitance $(\mathrm{Ct})$ change with powder particle size variation and 1 $\mathrm{kHz}$ square voltage: $\mathrm{He}$ (Vin: $5 \mathrm{~V}$ ) and $\mathrm{SF}_{6}(\mathrm{Vin}: 8 \mathrm{~V}$ ) (square: positive phase; circle: negative phase; hollow: He gas; filled: $\mathrm{SF}_{6}$ gas; the number 10 on the abscissa of the powder dia. represents the bare electrode).

voltage phase.

$\mathrm{Ct}$ is proportional to the quantity of charged particles transferred to the dielectric surface of the SDBD electrode, which corresponds to the degree of coverage of the plasma on the dielectric surface of the electrode. In all cases, the powder SDBD of the He gas, which has a lower breakdown voltage, had a wider plasma coverage area than that of the $\mathrm{SF}_{6}$ gas powder SDBD. Figure 13 shows the changes in $\mathrm{Ct}$ versus the particle size, which was measured from the Lissajous diagrams in Fig. 12. Ct of the He gas powder-SDBD decreased nearly linearly with the powder particle size. The He gas SDBD, which shows glow discharge at atmospheric pressure, covered the whole area of the SDBD electrode without spatial constrain; therefore, the He gas SDBD had the highest $\mathrm{Ct}$ with the no-powder electrode. However, when the powder was placed on the SDBD electrode, the glow discharge volume was constrained to the powder pores. Decreasing the size of the powder particle reduced the size of the pores that would be occupied by the plasma and $\mathrm{Ct}$ of the powder SDBD. When a less than $0.1 \mathrm{~mm}$ particle size powder was placed on the He gas SDBD, the glow discharge was transformed to filament discharge owing to the reinforced electric field in the pores and the spatial restriction of the discharge channels, which also reduced Ct. Conversely, the $\mathrm{SF}_{6}$ gas powder SDBD showed the highest $\mathrm{Ct}$ with the smallest powder particle size and a reverse relationship between Ct and the powder particle size, as shown in Fig. 13. The reinforced electric field in the smaller pores increased the discharge channel density on the edge of the HV electrode. Hence, the number density of the filament channel linearly increased with the number density of the pores. In addition, the filament channels with the length extension effectively filled the smaller pores, which were interconnected with each other. Therefore, $\mathrm{Ct}$ (i.e., the degree of plasma coverage) increased with the smaller powder in the $\mathrm{SF}_{6}$ powder SDBD. Consequently, the existence and size of the powder particle on an SDBD according to the gas species showed two opposite effects: a reduction of the plasma coverage on the SDBD electrode by the spatial constrain of the powder pores for He gas and an increase in the plasma coverage by the increment of the number density and length of the discharge channel in the pores for $\mathrm{SF}_{6}$ gas.

\section{Discussion}

Placing solid powder on an SDBD electrode covers some portion of the SDBD electrode surface with plasma, and after the discharge begins solid powder obstacles occupy the surface. Therefore, the small size of the powder particle implies that the portion of the plasma coverage area is small. Hence, for the He gas SDBD, which has a low breakdown voltage, the discharge on the electrode without powder has the highest $\mathrm{Ct}$ by covering the whole electrode area with a uniform glow discharge. Placing a solid powder reduces the portion of plasma coverage and lowers Ct. The effect of the particle size on the power consumption is smaller than that on $\mathrm{Ct}$ based on two possible reasons according to the current density change in the discharge channel. If the current density is not influenced by the powder particle size, the plasma volume remains by expanding to adjacent pores in the upper direction from the pores on the electrode surface. Second, because of the high electric field in the pores, the reduced total current is minimal, as the glow discharge volume changed to numerous small filament channels, each having a higher current density [10-12]. Based on the results of the changes in $\mathrm{Ct}$, the voltage to power consumption, and the current signals, both situations are plausible. Unlike the $\mathrm{He}$ gas, the $\mathrm{Ct}$ and consumed power increase as the powder particle size decreases in the $\mathrm{SF}_{6}$ gas powder SDBD. Because of the high electric field in the powder pores, especially in smaller pores, the length of the filament channel becomes longer, and the number of the filament seeds increases at the same time in the powder SDBD with $\mathrm{SF}_{6}$ gas, which has a strong short filament discharge at atmospheric pressure. Therefore, the power consumption at a given voltage increases as the particle size decreases in the $\mathrm{SF}_{6}$ gas case. In addition, $\mathrm{Ct}$ increases as the portion of the discharge increases in the smaller pores made by the smaller powder particles. The influence of the powder particle size on the powder $\mathrm{SDBD}$ is larger in $\mathrm{SF}_{6}$ gas than that in He gas; however, in all cases, the scale of the plasma generation volume and power consumption are larger in the He gas discharge. Therefore, an SDBD plasma in the gas having low breakdown voltage, such as He gas, can be used for the plasma surface treatment of a powder material whose particle size is in the range of $0.01 \sim 1 \mathrm{~mm}$. However, to treat a powder material with a particle size smaller than $0.01 \mathrm{~mm}$, the SDBD plasma with a gas with a high breakdown voltage would be more effective. In addition, if some gas species with a high breakdown voltage need to be activated by the plasma, it would be more effective to use a combination of SDBD plasma and porous dielectric media of smaller pores.

\section{Conclusions}

The characteristics of the SDBD of $\mathrm{He}$ gas and $\mathrm{SF}_{6}$ gas with alumina powder of a $0.01,0.1$, and $1 \mathrm{~mm}$ powder particle size on the SDBD electrode were evaluated. For the $0.5 \mathrm{~Hz}$ voltage powder SDBD, the discharge regime and properties of the filament discharge of the $\mathrm{He}$ and $\mathrm{SF}_{6}$ gas powder SDBDs were influenced by the alumina powder and particle size. When there was no powder on the electrode, the He gas SDBD showed a glow discharge covering almost all the electrode surfaces for both voltage polarities, and the $\mathrm{SF}_{6}$ gas $\mathrm{SDBD}$ showed numerous short filament channels in the positive voltage phase and few filament channels in the negative voltage phase. When alumina powder was placed on the SDBD electrode, the glow discharge current of the $\mathrm{He}$ gas powder SDBD changed to filament discharge as the powder particle size decreased. In the $\mathrm{SF}_{6}$ gas powder SDBD, the duration and number of pulse currents increased, and the different properties derived from the voltage polarity change disappeared 
as the powder particle size decreased. In addition, the voltage level where the first discharge current appeared was lowered by the smaller powder particle in the $\mathrm{SF}_{6}$ gas powder SDBD. However, in the He gas powder SDBD, lowering of the discharge start voltage level was not shown. The increased electric field in the small pores was more effective for the $\mathrm{SF}_{6}$ gas discharge than that of the He gas. Thus, the power consumption and total capacitance of the $1 \mathrm{kHz}$ voltage powder $\mathrm{SDBDs}$ of the $\mathrm{He}$ and $\mathrm{SF}_{6}$ gas showed different characteristics for the alumina powder. The total capacitance of the powder SDBD, i.e., the total size of the generated plasma with $1 \mathrm{kHz}$ voltage, decreased with the He gas as the powder particle size decreased; however, it increased with the $\mathrm{SF}_{6}$ gas. These results are caused by the spatial restriction of the discharge volume of the pores from the powder particles for the He gas discharge, which had a glow discharge with a large volume and no powder electrode. Conversely, the extension of the lengths of the filament channels and the increment of the filament population by the pores at the same time for the $\mathrm{SF}_{6}$ gas discharge, which had a short strong filament discharge with a no powder electrode. Therefore, solid powder can be used to increase the plasma coverage ratio; i.e., the total plasma volume, over the SDBD electrode with a gas of relatively high breakdown voltage, such as air, nitrogen, etc.

\section{Acknowledgements}

This work was supported by R\&D Program of 'Plasma Convergence \& Fundamental Research (code No. EN2021)' through the National Fusion Research Institute of Korea (NFRI) funded by the Government funds.

\section{References}

[1] S. H. Park and S. D. Kim, Colloids Surf. A: Physicochem. Eng. Aspects 133, 33 (1998).

[2] S. Put, C. Bertels, and A. Vanhulsel, Surf. Coat. Technol. 234, 76 (2013).

[3] Z. Szalay, K. Bodisova, H. Palkova, P. Svancarek, P. Durina, J. Rahel, A. Zahoranova, and D. Galusek, Ceram. Int. 40, 12737 (2014).

[4] H. Tanake, M. Kurihara, J. Y. Xu, N. Ohashi, S. Maruyama, Y. Moriyoshi, and T. Ishigaki, Thin Solid Films 506-507, 311 (2006).

[5] C. Arpagaus, G. Oberbossel, and P. R. von Rohr, Plasma Processes Polym. 15, 1800133 (2018).

[6] K. P. Singh and S. Roy, Appl. Phys. Lett. 91, 81504 (2007).

[7] F. O. Thomas, T. C. Corke, M. Iqbal, A. Kozlov, and D. Schatzman, Am. Inst. Aeronaut. Astronaut. 47, 9 (2009).

[8] J. Pons, E. Moreau, and G. Touchard, J. Phys. D: Appl. Phys. 38, 3635 (2005).

[9] L. Mangolini, K. Orlov, and U. Kortshagen, Appl. Phys. Lett. 80, $1722(2002)$.

[10] X. C. Li, L. F. Dong, and L. Wang, Chin. Phys. 14, 1418 (2005).

[11] X. Wang, C. Li, M. Lu, and Y. Pu, Plasma Source Sci. Technol. 12, 358 (2003).

[12] X. Li, D. Niu, and P. Jia, Int. Conf. Electr. Control Eng. 884 (2010).

[13] F. Massines and G. Gouda, J. Phys. D: Appl. Phys. 31, 3411 (1998).

[14] T. Namihira, D. Wang, S. Katsuki, R. Hackam, and H. Akiyama, IEEE Trans. Plasma Sci. 31, 1091 (2003).

[15] D. Wang, M. Jikuya, S. Yoshida, T. Namihira, S. Katsuki, and H. Akiyama, IEEE Trans. Plasma Sci. 35, 1098 (2007).

[16] H. Jiang, T. Shao, C. Zhang, W. Li, and P. Yan, IEEE Trans. Dielectr. Electr. Insul. 20, 1101 (2013).

[17] T. C. Manley, Trans. Electrochem. Soc. 84, 83 (1943).

[18] I. Biganzoli, R. Barni, A. Gurioli, R. Pertile, and C. Riccardi, J. Phys. Conf. Ser. 550, 012039 (2014). 